\title{
Exceptional long-term survivor (12 years) with metastatic gallbladder cancer
}

\author{
Franco Sacchetti ${ }^{1,2}$, Francesco Ardito ${ }^{1,2 *}$, Fabio M Vechio $^{3}$, Felice Giuliante ${ }^{1,2}$ \\ ${ }^{1}$ Hepatobiliary Surgery Unit, Fondazione Policlinico Universitario A. Gemelli IRCCS; Rome, Italy \\ ${ }^{2}$ Institute of Surgical Pathology, Università Cattolica del Sacro Cuore, Rome, Italy \\ ${ }^{3}$ Department of Pathology, Fondazione Policlinico Universitario A. Gemelli IRCCS; Rome, Italy
}

\begin{abstract}
We present a rare case of exceptional long-term survival (12 years) in a 78-year-old female patient presenting with stage IVb gallbladder carcinoma (GBC) with an unusual site of metastasis (left supraclavicular lymph node). After chemotherapy, a metabolic response of the metastatic regional and left supraclavicular lymph nodes was documented during the follow-up for 10 years. Because of biliary colic onset due to the presence of stones in the gallbladder, surgical resection was planned. In order to perform a curative resection, the patient underwent bi-segmentectomy $4 \mathrm{~b}-5$ en-bloc with the gallbladder + regional lymphadenectomy, 12 years after GBC diagnosis. At pathology, a G2 pT2 adenocarcinoma of the gallbladder was confirmed with pathologic response in all the 5 retrieved regional lymph nodes.
\end{abstract}

\section{Introduction}

Gallbladder carcinoma (GBC) is a rare aggressive cancer disease with poor prognosis. The clinical pessimism which surrounds this tumour is due to its late presentation and its lack of effective therapy. Indeed, most patients are diagnosed at an advanced stage of disease $[1,2]$. In patients with unresectable GBC the prognosis is dismal and palliative support is given in order to control morbidity resulting from biliary obstruction, pain, cachexia and infections. In such patients the median survival is 6 months with a 5 -year overall survival rate $<10 \%$ $[1,2]$. Only $25 \%$ of patients will undergo potentially curative surgery, and about $16 \%$ will survive for more than 5 years [3].

We present a rare case of exceptional long-term survival (12 years) in a 78-year-old female patient presenting with an asymptomatic metastatic GBC.

\section{Case presentation}

We report the case of a 78-year-old female patient in good clinical conditions, with a history of hepatitis $\mathrm{C}$ virus infection, arterial hypertension on drug therapy, paroxysmal atrial fibrillation and Helicobacter pylori negative chronic gastritis.

Twelve years before referral to our Unit, at the age of 66 years, an incidental finding of focal thickening of the gallbladder wall at the fundus, was documented at abdominal ultrasound performed for dyspepsia. The ultrasound also showed a mobile endoluminal stone with a diameter of $17 \mathrm{~mm}$. The focal thickening of the gallbladder wall was confirmed at abdominal CT scan. The finding was strongly suspicious for GBC.

The patient underwent Positron Emission Tomography-CT (PET-CT) examination with 18fludeoxyglucose (18F-FDG) which documented, in the portal and late phases, discrete metabolic activity (SUV-max $=5.6)$ at the level of the thickened wall of the gallbladder fundus. Discrete metabolic activity was also visible at the level of the hepatic hilum where a pathological lymph node package of about 3 $\mathrm{cm}$ in diameter was documented. An additional pathological lymph node of $1.7 \mathrm{~cm}$ in diameter with weaker metabolic activity in the left supraclavicular region was documented (Figure 1).

Fine-needle aspiration for cytological examination was performed on the left supraclavicular lymph node resulting in Cytokeratin 7 positive adenocarcinoma localization (Figure 2).

The patient presented an asymptomatic metastatic GBC, classified as stage IVb according to the 8th Edition of the American Joint Committee on Cancer (AJCC) Staging Manual [4]. The patient underwent palliative chemotherapy. First, Gemcitabine monotherapy was administered intravenously for 3 months. Later, Capecitabine 500 mg was undertaken for 2 years.

After chemotherapy a new PET-CT examination with $18 \mathrm{~F}-\mathrm{FDG}$ did not document any area with significant metabolic activity in the body, to be referred to the presence of active neoplastic disease (Figure 3). After stopping chemotherapy, the patient underwent regular radiological follow-up with PET-CT examination with 18F-FDG every year for 10 years, without evidence of disease activity at the local or metastatic level.

Twelve years after metastatic GBC diagnosis, the patient was referred at our Unit because of recurrent biliary colic with abdominal pain in the right upper quadrant. Blood chemistry was normal. Tumour markers were normal. In particular, the carcinoembryonic antigen (CEA) level was $1.4 \mathrm{ng} / \mathrm{mL}$ (n.v. <5.0) and the carbohydrate antigen 19-9 (CA 19-9) level was $25.2 \mathrm{UI} / \mathrm{mL}$ (n.v. <37.0).

${ }^{\star}$ Correspondence to: Francesco Ardito, Hepatobiliary Surgery Unit, Catholic University of the Sacred Heart, L.go A. Gemelli, Rome - Italy, Tel: +39-0630155626, E-mail: francesco.ardito@unicatt.it

Received: October 25, 2019; Accepted: November 25, 2019; Published: November 29, 2019 
Thoracic-abdominal CT scan was performed. At the abdominal level, the examination confirmed asymmetric thickening of the anterior wall of the gallbladder's body without radiological signs of invasion of the surrounding liver parenchyma. A suspicious roundish lymph node with a short axis of $11 \mathrm{~mm}$ in the perigallbladder region was also described (Figure 4).

In relation to the very long patient's oncologic history (12 years after GBC diagnosis and 10 years of persistent metabolic response of the metastatic tumour), in relation to the onset of clinical symptomatology due to the presence of a stone in the gallbladder and in relation to the good clinical conditions of the patient, surgical resection was planned.

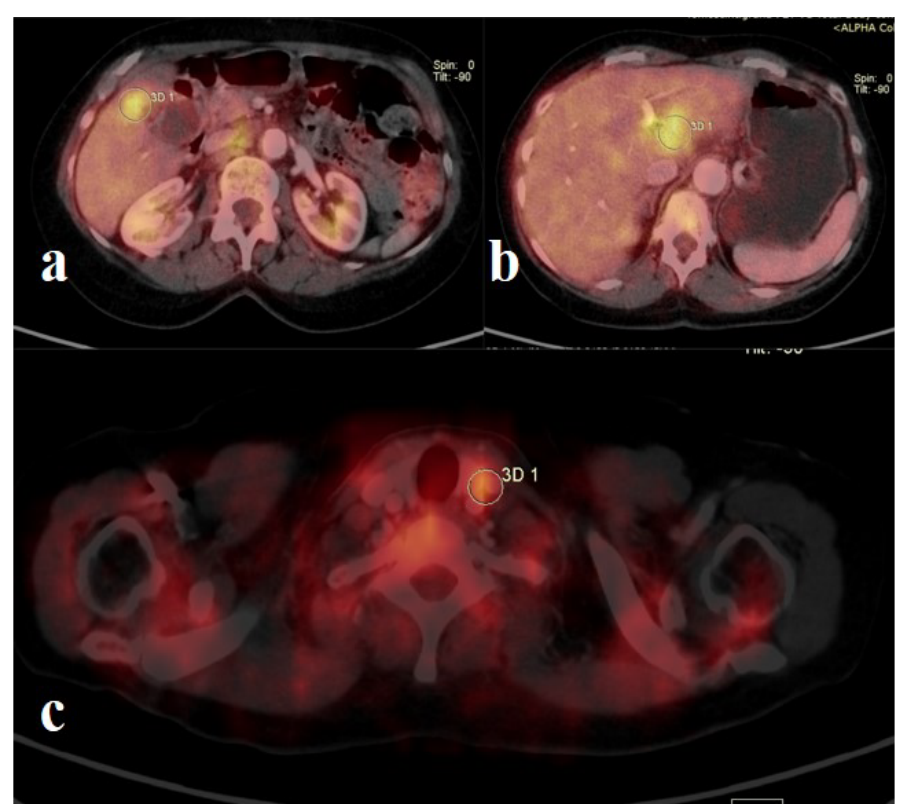

Figure 1. PET-CT with $18 \mathrm{~F}-\mathrm{FDG}$ performed at the time of GBC diagnosis. Metabolic activity (SUV-max $=5.6$ ) was documented at the level of the thickened wall of the gallbladder fundus (a), Discrete metabolic activity was also visible at the level of the hepatic hilum (b) where a pathological lymph node package of about $3 \mathrm{~cm}$ in diameter was documented. An additional pathological lymph node of $1.7 \mathrm{~cm}$ in diameter with weaker metabolic activity in the left supraclavicular region was documented (c)

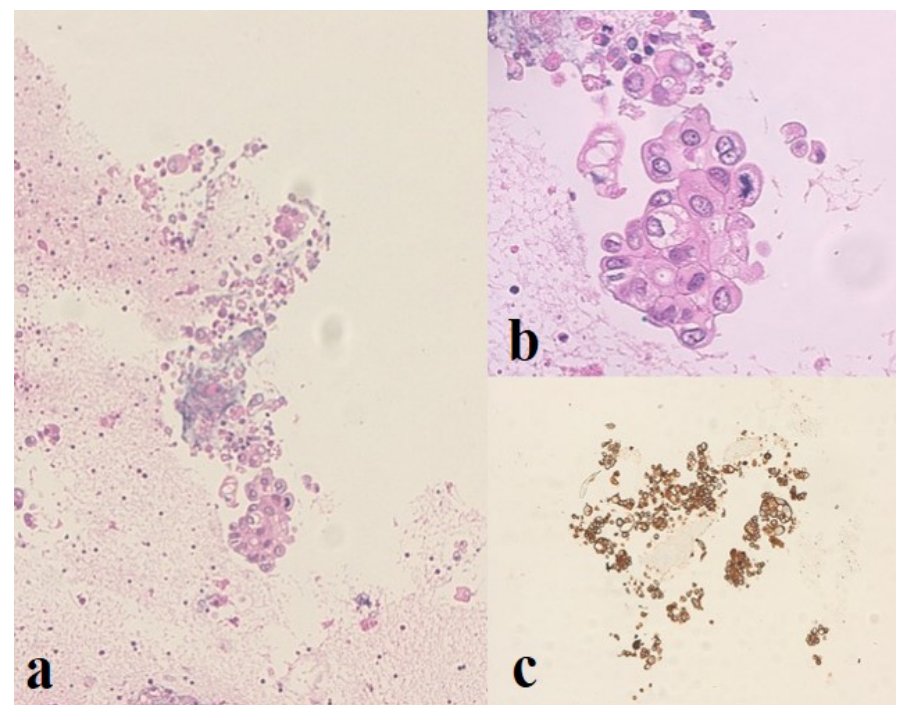

Figure 2. Cytological examination of the left supraclavicular lymph node before starting chemotherapy. The cytological features of the lesion are characterized by epithelial cells with irregular nuclei and eosinophilic cytoplasm (a-b). Neoplastic cells show marked immunoreactivity for Cytokeratin 7 (c). Diagnosis: adenocarcinoma localization.

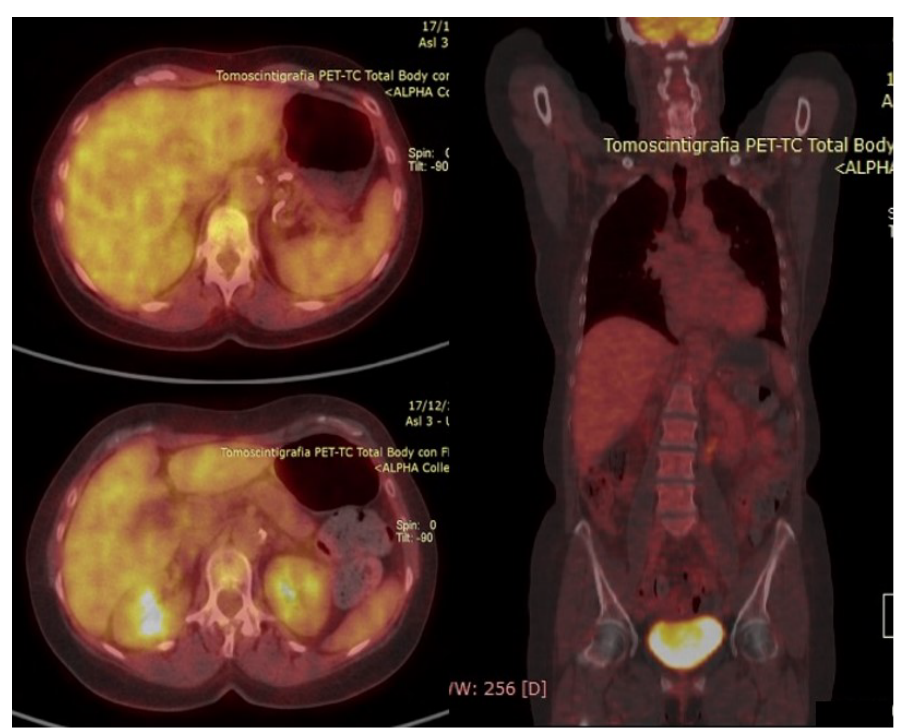

Figure 3. PET-CT with $18 \mathrm{~F}-\mathrm{FDG}$ after chemotherapy: no area with significant metabolic activity in the body, to be referred to the presence of active neoplastic disease, was documented.

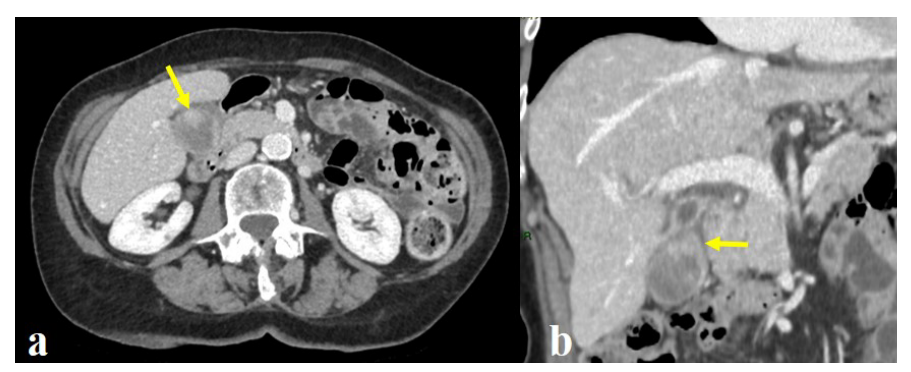

Figure 4. Abdominal CT scan 12 years after metastatic GBC diagnosis. At the axial view (a) and at the coronal view (b) an asymmetric thickening of the anterior wall of the gallbladder's body was confirmed (yellow arrow).

The patient underwent hepatic bi-segmentectomy $4 \mathrm{~b}-5$ en-bloc with the gallbladder + regional lymphadenectomy (excision of lymph nodes around the hepatic pedicle, around the common hepatic artery and in the superior retro-pancreatic region). A fresh frozen section of the cystic duct stump was sent for histologic examination and did not show any neoplastic invasion.

The specimen confirmed the presence of an exophytic mass at the level of the gallbladder body (Figure 5). At pathology a moderately differentiated adenocarcinoma of the gallbladder $(5.5 \mathrm{~cm}$ in diameter) invading the muscle layer and the perimuscular connective tissue, without extension into the liver (Figure 6) was documented. Five regional lymph nodes (diameter ranging from 1.5 to $3 \mathrm{~cm}$ ) were negative for tumour localization.

KRas and NRas mutations were assessed in DNA of the neoplastic specimen (codons 12, 13, 59, 61, 117 and 146). No Ras mutations were found. Postoperative course was uneventful, and the patient was discharged in good general conditions on postoperative day 5 .

\section{Discussion}

Our manuscript showed an exceptional long-term survival (12 years) in a patient with metastatic GBC, treated by chemotherapy alone. Despite improvements in diagnostic modalities, most of GBCs are diagnosed at an advanced stage [5]. 


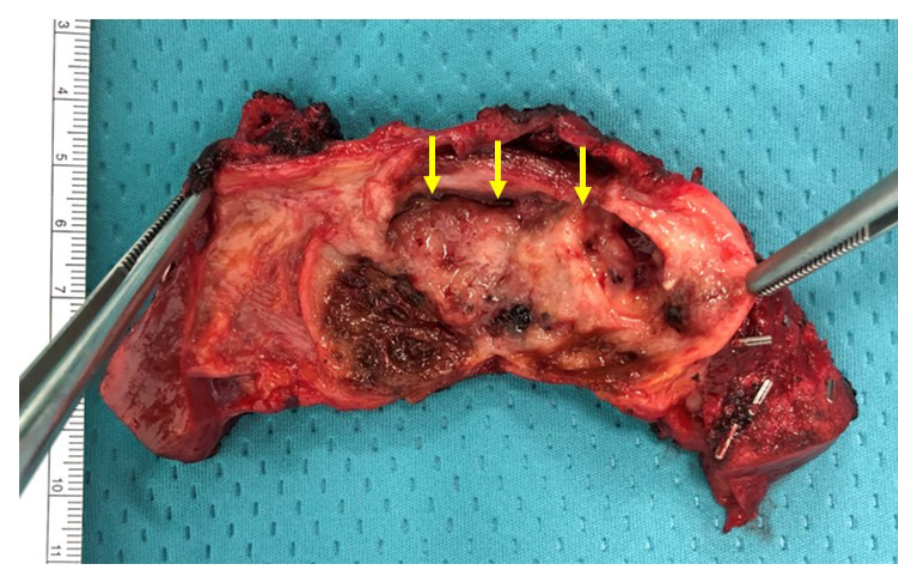

Figure 5. The specimen confirmed the presence of an exophytic mass at the level of the gallbladder body (yellow arrow).
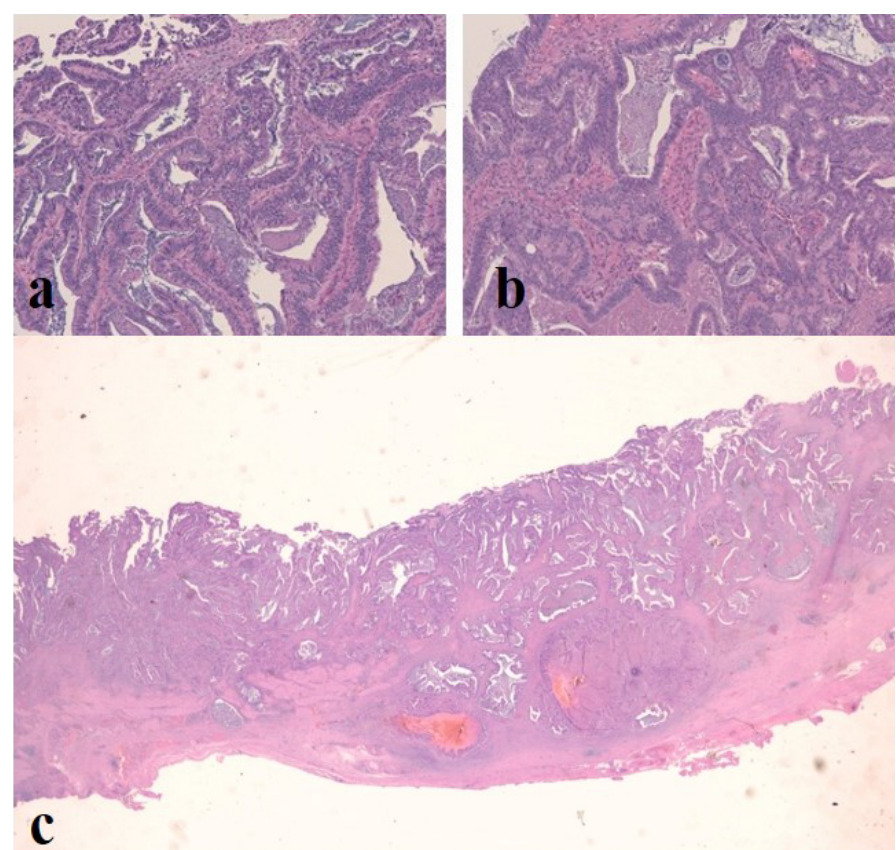

Figure 6. Pathological examination of the specimen: moderately differentiated adenocarcinoma of the gallbladder $(5.5 \mathrm{~cm}$ in diameter) invading the muscle layer and the perimuscular connective tissue, without extension into the liver. Tumor cells show a tubulo-glandular pattern with a focally dilated glandular lumen (a-b). At a panoramic view it is evident the absence of a polypoid pattern (c).

The first interesting point of our report was the site of metastasis of GBC: the left supraclavicular lymph node. In general, the nodal involvement in GBC includes stations from the hepatoduodenal ligament toward the nodes around the head of the pancreas [3]. The regional lymph nodes of the gallbladder include the cystic duct, pericholedochal, hilar (hepatoduodenal ligament), periarterial, periportal, common hepatic artery and superior retro-pancreatic nodes [3]. After spreading to the periportal lymph nodes, GBC may spread to the aorto-caval station, but may also cross to the coeliac nodal station before advancing to more distant sites [3]. Para-aortic lymph nodes are considered as distant metastases.

Distant left supraclavicular lymph node metastasis has never been reported in the Western experience. However, the incidence of GBC is low in North America, but is high in countries like India, Chile, and Japan [6]. Indeed, in a paper by Haribhakti et al. [7] from India, 12 cases of left supraclavicular lymph node metastasis were reported.
The second interesting point of our report was the exceptional long-term survival of the unresected patient: 12 years. Chemotherapy by the combination of gemcitabine and capecitabine is considered as the standard first-line therapy for advanced GBC $[6,8]$. However, prognosis in such unresected patients is poor with consequent median progression-free survival of 6 to 9 months and overall survival of 9.8 to 14 months [9-12]. The patient in our manuscript was considered as unresectable patient in another hospital and she underwent palliative treatment by Gemcitabine-based doublet chemotherapy for 2 years. At radiologic imaging after chemotherapy a persistent metabolic response was documented for 10 years, without any sign of tumour progression.

The patient was referred to our Unit 12 years after GBC diagnosis because of recurrent biliary colic due to the presence of stones in the gallbladder.

The role of surgery in stage IV GBC is not well established. In general, no survival benefit is documented after aggressive surgery in patients with involved para-aortic, coeliac or superior mesenteric artery nodes [3]. However, in the literature, the possibility of long-term survival after radical surgery in patients with metastatic GBC has been described [5]. Some Eastern surgical experiences showed that patients with advanced GBC can benefit from surgical resection even when para-aortic lymph node metastases or single liver metastasis are present $[13,14]$. In the paper by Nishio et al. [14] the 5-year overall survival rate was $12 \%$ with an overall hospital mortality of $14 \%$. In this series patients with para-aortic lymph node metastases or with single liver metastasis who underwent curative resection, survived longer than those with other distant metastasis or those with unresectable advanced cancer [13]. Of course, these results come from small single centre series and surgical indications should be determined on an individual basis, based on clinical status.

Indication for aggressive surgery in our patient was determined by the very long-term metabolic response of the tumour, by her good clinical conditions and by the onset of recurrent biliary colic.

The third interesting point of our manuscript was the pathology report: complete pathologic response in all the retrieved regional lymph nodes. These lymph nodes, as the left supraclavicular lymph node, showed metabolic activity at PET-CT before chemotherapy and they have to be considered as neoplastic lymph nodes with complete pathologic response.

\section{Conclusion}

In conclusion the particularity of the proposed clinical case concerns the exceptional long-term survival (12 years) of a patient with stage IVb GBC with an unusual site of metastasis (left supraclavicular lymph node). After chemotherapy a metabolic response of the metastatic lymph nodes was documented that resulted in a complete pathologic response following curative resection.

\section{References}

1. Coburn NG, Cleary SP, Tan JC, Law CH (2008) Surgery for gallbladder cancer: a population-based analysis. J Am Coll Surg 207: 371-382. [Crossref]

2. Hundal R, Shaffer EA (2014) Gallbladder cancer: epidemiology and outcome. Clin Epidemiol 6: 99-109. [Crossref]

3. Aloia TA, Járufe N, Javle M, Maithel SK, Roa JC, et al. (2015) Gallbladder cancer expert consensus statement. HPB (Oxford) 17: 681-690. [Crossref]

4. Chun YS, Pawlik TM, Vauthey JN (2018) 8th Edition of the AJCC Cancer Staging Manual: Pancreas and Hepatobiliary Cancers. Ann Surg Oncol 25: 845-847. [Crossref] 
5. Kang MJ, Song Y, Jang JY, Han IW, Kim SW (2012) Role of radical surgery in patients with stage IV gallbladder cancer. HPB (Oxford) 14: 805-811. [Crossref]

6. Zaidi MY, Maithel SK (2018) Updates on Gallbladder Cancer Management. Curr Oncol Rep 20: 21. [Crossref]

7. Haribhakti SP, Awasthi S, Pradeep R, Kapoor VK, Kaushik SP (1977) Carcinoma gallbladder: atypical presentations and unusual associations. Trop Gastroenterol 18: 32-34. [Crossref]

8. Iyer RV, Pokuri VK, Groman A, Ma WW, Malhotra U, et al. (2018) A Multicenter Phase II Study of Gemcitabine, Capecitabine, and Bevacizumab for Locally Advanced or Metastatic Biliary Tract Cancer. Am J Clin Oncol 41: 649-655. [Crossref]

9. Knox JJ, Hedley D, Oza A, Feld R, Siu LL, et al. (2005) Combining gemcitabine and capecitabine in patients with advanced biliary cancer: a phase II trial. J Clin Oncol 23 : 2332-2338. [Crossref]
10. Cho JY, Paik YH, Chang YS, Lee SJ, Lee DK, et al. (2005) Capecitabine combined with gemcitabine (CapGem) as first-line treatment in patients with advanced/metastatic biliary tract carcinoma. Cancer 104: 2753-2758. [Crossref]

11. Iyer RV, Gibbs J, Kuvshinoff B, Fakih M, Kepner J, et al. (2007) A phase II study of gemcitabine and capecitabine in advanced cholangiocarcinoma and carcinoma of the gallbladder: a single-institution prospective study. Ann Surg Oncol 14: 3202-3209. [Crossref]

12. Koeberle D, Saletti P, Borner M, Gerber D, Dietrich D, et al. (2008) Patient-reported outcomes of patients with advanced biliary tract cancers receiving gemcitabine plus capecitabine: a multicenter, phase II trial of the Swiss Group for Clinical Cancer Research. J Clin Oncol 26: 3702-3708. [Crossref]

13. Shimizu H, Kimura F, Yoshidome H, Ohtsuka M, Kato A, et al. (2007) Aggressive surgical approach for stage IV gallbladder carcinoma based on Japanese Society of Biliary Surgery classification. J Hepatobiliary Pancreat Surg 14: 358-365. [Crossref]

14. Nishio H, Nagino M, Ebata T, Yokoyama Y, Igami T, et al. (2007) Aggressive surgery for stage IV gallbladder carcinoma; what are the contraindications? J Hepatobiliary Pancreat Surg 14: 351-357. [Crossref]

Copyright: (C2019 Sacchetti F. This is an open-access article distributed under the terms of the Creative Commons Attribution License, which permits unrestricted use, distribution, and reproduction in any medium, provided the original author and source are credited. 